\title{
Formulación de los objetivos específicos desde el alcance correlacional en trabajos de investigación
}

\author{
José Luis Arias Gonzáles \\ joseariasgon6@gmail.com \\ Mitsuo Roger Covinos Gallardo \\ covinosmitsuo.905@gmail.com \\ Milagros Cáceres Chávez \\ milagros_c1@hotmail.com
}

\section{RESUMEN}

El presente estudio tuvo como objetivo principal analizar como formular los objetivos específicos desde el alcance correlacional, se realizó un estudio documental de corte transversal, el universo se constituyó por los libros de investigación científica y artículos científicos alineados al tema de estudio y se utilizó el método no probabilístico para hallar la muestra que se constituyó por 22 libros de investigación científica y artículos científicos, la técnica utilizada fue el análisis de documentos y se utilizó la ficha bibliográfica como instrumento para reunir y analizar la información. Los resultados permiten concluir que, la forma idónea de formular los objetivos específicos desde el alcance correlacional es por variables o por dimensiones, se deben plantear con verbos exploratorios o descriptivos en infinitivo, no deben ser objetivos explicativos o proyectivos (de mayor nivel que el objetivo general), ni deben parecer tareas o actividades que quiere cumplir el investigador.

Palabras clave: Correlacional; objetivos específicos; alcances de investigación; objetivos de investigación 


\title{
Formulation of specific objectives from the correlational scope in research paper
}

\begin{abstract}
:
The main objective of the present study was to analyze how to formulate the specific objectives from the correlational scope, a cross-sectional documentary study was carried out, the universe was constituted by scientific research books and scientific articles aligned to the study topic and the method was used Non-probabilistic to find the sample that consisted of 22 scientific research books and scientific articles, the technique used was the analysis of documents and the bibliographic record was used as an instrument to gather and analyze the information. The results allow us to conclude that the ideal way to formulate the specific objectives from the correlational scope is by variables or by dimensions, they should be raised with exploratory or descriptive verbs in the infinitive, they should not be explanatory or projective objectives (of a higher level than the objective general), nor should they appear to be tasks or activities that the researcher wants to accomplish.
\end{abstract}

Keywords: Correlational; specific objectives; research scopes; investigation objectives

Artículo recibido: 03. agosto. 2020 Aceptado para publicación: 11. setiembre. 2020 Correspondencia: joseariasgon6@gmail.com Conflictos de Interés: Ninguna que declarar 


\section{INTRODUCCIÓN}

Formular los objetivos de investigación representa una las dificultades que la mayoría de estudiantes e investigadores atraviesan al momento de redactar su trabajo de investigación dado su importancia y función crucial, es importante reconocer todos los pasos, procesos y métodos para redactar objetivos que se puedan lograr y se alineen adecuadamente con el estudio, asimismo, no existen estudios que identifiquen concretamente como se deben formular los objetivos específicos de acuerdo al alcance correlacional., es por ello que, el presente artículo científico se realiza con el principal objetivo de analizar como formular los objetivos específicos desde el alcance correlacional, para eso, es fundamental entender, en primera instancia, qué es un objetivo de investigación.

Según Bastidas (2019), los objetivos de investigación deben ser claros, realistas y alcanzables, asímismo, el objetivo de investigación debe tener un verbo en infinitivo que va a indicar la acción, un evento que refiere el estudio de las variables y unidades de estudio que pueden ser personas, objetos, instituciones, o un contexto que refiere un lugar donde se realiza el estudio. Para Ocegueda (2004), Los objetivos de investigación tienen la principal función de definir lo que se quiere cumplir, como todo comienza con un problema y es algo que necesita ser atendido, el objetivo es aquella acción para atender el problema, del mismo modo, los objetivos tienen la función de indagar e investigar acerca del problema, más no de resolverlos, al respecto Flórez y Tobón (2013), precisan que los objetivos se relacionan con los conocimientos que el investigador pretende alcanzar y con las preguntas realizadas para el problema del estudio. En concordancia a los dicho por estos autores, se establece que los objetivos de investigación constituyen un componente relevante y central en todo el proceso de la investigación.

\subsection{ALCANCE CORRELACIONAL}

Existen diveros alcances de investigación que permiten indagar sobre un problema o una situación problemática, desde lo exploratorio donde las bases teóricas son escasas (Salinas y Cárdenas, 2009), hasta los explicativos en el que se puede manipular deliberdamante la variable independiente para buscar un resultado o efecto sobre la variable dependiente (Zurita-Cruz, Márquez-Gonzáles, Miranda-Novales, y VillasisKeever, 2018), el alcance correlacional se presenta en un punto medio y tiene como finalidad medir el grado de asociación y/o relación entre dos variables o categorías desde 
un enfoque cuantitativo (Hernández-Sampieri y Mendoza, 2018), en tal sentido, un ejemplo claro para plantear un objetivo general sería: Determinar la relación entre la la obesidad y el riesdo de paceder diabetes en adultos mayores de 60 años. Para Cabezas, Andrade y Torres (2018), un alcance correlacional, también llamado nivel correlacional, tiene la finalidad de evaluar la relación que existe entre dos variables mediante la aplicación de técnicas estadísticas, así mismo, mediante la medición de una variable se va a conocer como se comporta la otra variable; Tamayo (2003), menciona que, en los estudios correlacionales no es posible el control experimental, no direcciona a establecer una causalidad entre variables, sin embargo, es un primer paso para empezar a identificar causa-efecto, estas teorías son similiares a lo propuesto por Cazau (2006), quien menciona que, la finalidad de este tipo de estudio es establecer concretamente si hay o no correlación entre dos variables, además de establecer su grado de intensidad. A continuación se presenta una tabla comparativa para establecer las diferencias entre el alcance correlacional y los demás alcances.

Tabla 1. Comparativo de los alcances de investigación

\begin{tabular}{ll}
\hline ALCANCE & PROPÓSITO \\
\hline Exploratorio & Se estudia un tema poco abordado de una \\
& variable \\
Descriptivo & Observa, describe y fundamenta aspectos \\
& de una variable \\
Correlacional & Mide el grado de relacion entre dos \\
& variables \\
Explicativo & Establece causa efecto entre dos o más \\
& variables, se manipula la variable \\
& independiente.
\end{tabular}

Fuente: Investigación y Comunicación (Dankhe, 1986)

La tabla muestra que existe una diferencia conceptual y de propósito entre los cuatro alcances presentados, según Rios (2017), estos cuatro tipos de investigación se diferencian por la teoría que existe acerca de las variables o el feonómeno, y hasta que instancia del estudio desea llegar el investigador. 


\subsection{OBJETIVOS ESPECÍFICOS}

Los objetivos específcos se pueden formular de distintas formas, dependiendo del alcance de investigación, el diseño y la situación problemática del estudio, la forma correcta de redactar los objetivos es siguiendo un órden lógico que se alinea con el desarrollo del trabajo de investigación. Para Caballero (2014), los objetivos específicos "son enunciados propocionales desentrañados en etapas de un objetivo general" (p. 149), deben ser: Cualitativos, conductuales y específicos, Behar (2008), refiere que, la cantidad de los objetivos específicos que se formulen depende de lo que se necesita para lograr el objetivo de investigación o están orientados a lograr cada etapa del desarrollo de la investigación, similar idea tiene Bernal (2006), los objetivos específicos son las etapas o pasos que se deben realizar para lograr el objetivo general; por otro lado, Barragán, Salman, Aillón, Córdova, Langer, Sanjinés y Rojas (2011), mencionan que, se debe evitar disgregar los objetivos específicos del objetivo general y que estos deben relacionarse con el objetivo general por medio de sus dimensiones.

Bustamante y Mendoza (2013), precisan que las preguntas de investigación deben establecer los valores a medir para cada variable de estudio, por dicha razón, dado que, los objetivos específicos deben responder directamente a las preguntas específicas, entonces, en el alcance correlacional la presentación de los objetivos se realiza por variables, para Arias (2012), los objetivos específicos “indican con precisión los conceptos, variables o dimensiones que serán objeto de estudio, se derivan del objetivo general y contribuyen al logro de este" (p. 45). Balestrini (2006) menciona que, se trata de disgregar los elementos o dimensiones del problema o del objetivo general y además de limitar lo que se desea estudiar, teoría que coincide por Cruz, Olivares, y Gonzáles (2014), quienes precisan que, "los objetivos específicos se desprenden del general y son pasos orientados al logro de este" (p. 95)

Por otro lado, Hurtado (2005), menciona que, los objetivos específicos desde el alcance correlacional estan conformados por estadíos que se preestablecieron por el objetivo general y por ningún motivo deben ser de mayor nivel que este. Se debe tener cuidado con igualar los objetivos de investigación con tareas y actividades, la característica de toda investigaicón científica es lograr un conocimiento por medio del método científico, por tal razón, un objetivo de investigación debe buscar el conocimiento científico, en 
cambio, una tarea es lo que se va a realizar para llevar a cabo los objetivos; para Hurtado, (2005), las tareas y actividades estan implícitas en el desarrollo de la investigación y no se deben considerar como objetivos de investigación, un ejemplo claro de una tarea es: Elegir una muestra para la investigación

Con respecto a los verbos que deben tener los objetivos específicos, según Arias (2017), se presenta la siguiente tabla de verbos según el alcance de investigación:

Tabla 2. Verbos de investigación según el alcance

\begin{tabular}{llll}
\hline EXPLORATORIO & DESCRIPTIVO & CORRELACIONAL & EXPLICATIVO \\
\hline Conocer & Calcular & Establecer & Comprobar \\
Definir & Medir & Determinar & Demostrar \\
Indagar & Clasificar & Precisar & Evaluar \\
Sondear & Comparar & Analizar & Verificar \\
Explorar & Identificar & Medir & Determinar \\
Analizar & Cuantificar & Relacionar & Establecer \\
Comprender & Establecer & Valorar & Explicar \\
\hline
\end{tabular}

Fuente: Adaptación del libro Mitos y errores en la elaboración de tesis y proyecto de investigación, (Arias, 2017).

\section{MATERIALES Y MÉTODOS}

La metodología utilizada para este artículo científico fue basada en un tipo de investigación documental, según Creswell (2014), estos estudios se caracterizan por realizar consultas e indagaciones en documentos de los cuales se obtienen los resultados que el investigador busca. Es un estudio de corte transversal, según Kerlinger y Lee (2001), la característica principal de estos estudios es que se hacen en una sola instancia, por ello, no existe un seguimiento.

El universo estuvo constituido por los libros de investigación científica y artículos científicos alineados al tema de estudio, y para la muestra se utiliza el método no probabilístico, según Hernández-Sampieri y Mendoza (2018), el método no probabilístico se utiliza cuando se eligen sub grupos de la población representativos a criterio del 
investigador y todos los sujetos tienen la misma probabilidad de ser elegidos, por lo cual, la muestra se conformó por 22 libros de investigación científica y artículos científicos.

La técnica utilizada fue el análisis de documentos, según Creswell (2014), esta técnica permite al investigador indagar sobre bases docuementales y obtener información sobre ellas, el instrumento utilizado fue la ficha bibliográfica el cual permite registrar, ordenar y resumir la información de fuentes bibliográficas.

Para procesar la información se procedió a resumir y reunir la información acerca de los objetivos de investigación, objetivos específicos, verbos de investigación, alcances de investigación para luego realizar un análisis crítico según las diferentes teorías presentadas por los autores para la presentación de los resultados.

\section{RESULTADOS Y DISCUSIÓN}

Como se ha presentado líneas arriba, según los autores y las bases teóricas, el fin de los estudios con alcance correlacional es asociar o relacionar las variables del estudio y los objetivos específicos se dervian del obetivo general por medio de la disgregación de sus variables y/o dimensiones o se pueden presentar por etapas, por otro lado, al formular los objetivos específicos se debe tener en cuenta que estos no pueden ser de igual o mayor alcance que el objetivo general, en tal sentido, los objetivos específicos desde el alcance correlacional deben tener las siguientes características

a) Deben ser objetivos con verbos exploratorios o descriptivos

b) Los objetivos específicos pueden tener verbos descriptivos o exploratorios diferentes.

c) Empezar con un verbo en infinitivo

d) Cada objetivo debe tener solo un verbo

e) No deben ser objetivos explicativos o proyectivos (de mayor nivel que el objetivo general)

f) No deben parecer tareas o actividades que quiere cumplir el investigador

g) Se pueden formulan por dimensiones o variables

h) Se pueden formulan por etapas

Para Bustamante y Mendoza (2013), Arias (2012), Balestrini (2006), y Cruz, Olivares, y Gonzáles (2014), se pueden formular los objetivos específicos de la siguiente forma: 
a) Por variables

- Objetivo específico 1: Variable 1

- Objetivo específico 2: Variable 2

b) Por dimensiones

- Objetivo específico 1: Dimensión 1 de la variable 1

- Objetivo específico 2: Dimensión 2 de la variable 1

- Objetivo específico 3: Dimensión 1 de la variable 2

- Objetivo específico 4: Dimensión 2 de la variable 2

Si el título pretende determinar la relación entre el clima laboral y la autoestima de los trabajadores de una empresa los objetivos específicos podrían ser los siguientes:

a) Por variables:

- Describir el clima laboral en la empresa (Variable 1)

- Medir la autoestima de los trabajadores (Variable 2)

b) También pueden ser presentados por dimensiones:

- Analizar la comunicación entre los trabajadores (Clima laboral)

- Medir el índice de participación de los trabajadores (Clima laboral)

- Conocer la autoestima social de los trabajadores (Autoestima)

- Identificar la autoestima familiar de los trabajadores (Autoestima)

Otros autores como Caballero (2014), Bernal (2006) y Behar (2008), precisan que los objetivos específicos deben ser presentados por etapas y que estan orientadas al logro de cada etapa o capítulo de la investigación en secuencia, se presentan de la siguiente forma:

1. Marco teórico:

2. Situación problemática:

3. Metodología

4. Resultados que se pretenden obtener

5. Propuesta de solución

Los objetivos específicos serían: 
1. Indagar sobre las bases teóricas

2. Describir las variables de interés

3. Precisar la metodología a utilizar de acuerdo con el estudio

4. Identifcar las deficiencias o situaciones que se han descrito

5. Proponer soluciones para las deficiencias encontradas en el estudio

Barragán, et al (2011), diferen de las teorías antes presentadas; los autores precisan que los objetivos específicos no se deben disgregar del objetivo general y más bien, estos deben guardar relación directa con el objetivo general, a continuación se presenta un ejemplo:

- Objetivo específico 1: Variable 1 con dimensión 1

- Objetivo específico 2: Variable 1 con dimensión 2

- Objetivo específico 3: Variable 1 con dimensión 3

Si el título pretende medir la relación entre los estilos de liderazgo y la productividad de los trabajadores de una empresa, los objetivos específicos se presentan de la siguiente forma:

- Medir la relación entre los estilos de liderazgo y el nivel de eficiencia de los trabajadores

- Establecer la relación entre los estilos de liderazgo y el nivel de eficacia de los trabajadores

- Identificar la relación entre los estilos de liderazgo y el nivel de efectividad de los trabajadores

\section{CONCLUSIÓN O CONSIDERACIONES FINALES}

Se han presentado tres formas diferentes en las que se pueden formular los objetivos específicos desde el alcance correlacional: Por variables o dimensiones, por etapas y por relación directa a la variable, sin embargo, sosteniendo lo dicho por Bustamante y Mendoza (2013), Arias (2012), Balestrini (2006), Cruz, Olivares, y Gonzáles (2014), Hernández-Sampieri y Mendoza (2018), Cabezas, Andrade y Torres (2018), y Cazau (2006), la principal característica del alcance correlacional es la relación o asociación de las variables, y los objetivos específicos pueden disgragarse en dimensiones del objetivo 
general o se formulan precisando de las variables, se concluye que, la forma idónea de presentar los objetivos específcos desde el alcance correlacional es por variables o por dimensiones.

\section{LISTA DE REFERENCIAS}

Arias, F. (2012). El proyecto de investigación (6ta ed.). Caracas: Episteme.

Arias, F. (2017). Mitos y errores en la elaboración de tesis y proyecto de investigación (2da ed.). Caracas: Episteme.

Balestrini, M. (2006). Como se elabora el proyecto de investigación (7ma ed.). Caracas: Consultores asociados.

Barragán, R., Salman, T., Aillón, V., Córdova , J., Langer, E., Sanjinés, J., y Rojas, R. (2011). Guía para la formulación y ejecución de proyectos de investigación (4ta ed.). La Paz: Fundación PIEB.

Bastidas, A. (2019). Fundamento para la redacción de objetivos en los trabajados de investigación de pregrado. Mextesol, 43(1).

Bernal, C. (2006). Metodología de la investigación (2da ed.). México: Pearson.

Bustamante, G., y Mendoza, C. (2013). Estudios de correlación. Actualización clínica, 33, 1690-1694.

Caballero, A. (2014). Metodología integral innovadora para planes y tesis. México D.F.: Cerigage Learning Editores.

Cabezas, E., Andrade, N., y Torres , J. (2018). Introducción a la metodología de la investigación científica (1ra ed.). Ecuador: Universidad de las Fuerzas Armadas.

Cazau, P. (2006). Introducción a la investigación en Ciencias Sociales (3ra ed.). Buenos Aires.

Creswell, J. (2014). Research Design (4ta ed.). United Kingdom: Sage publications.

Cruz, C., Olivares, S., y Gonzáles, M. (2014). Metodología de la investigación. México: Grupo editorial Patria. 
Dankhe, G. (1986). Investigación y comuncación. Madrid: Mc Graw Hill.

Flórez, R., y Tobón, A. (2003). Investigación educagtiva y pedagógica. Bogotá: Mc Graw Hill.

Hernández-Sampieri, R., y Mendoza, C. (2018). Metodología de la investigación, las rutas cuantitativa. cualitativa y mixta. Ciudad de México: Mc Graw Hill.

Hurtado, J. (2005). Como formular objetivos de investigación. Caracas: Fundación Sypal.

Kerlinger, F., y Lee, H. (2001). Investigación del comportamiento, metodo de investigación en las Ciencias Sociales (3ra ed.). México D.F.: Mc Graw Hill.

Ocegueda, C. (2004). Metodología de la investigación (2da ed.). Albox.

Rios, R. (2017). Metodología para la investigaicón y redacción (1ra ed.). Málaga: Servicios académicos intercontinentales.

Salinas, P., y Cárdenas, M. (2009). Métodos de investigación social (2da ed.). Quito: Quipus.

Tamayo, M. (2003). El proceso de la investigaciuón científica (4ta ed.). México D.F.: Lmusa, S.A.

Zurita-Cruz, J., Márquez-Gonzáles, H., Miranda-Novales, G., y Villasis-Keever, M. (2019). Estudios experimentales: Diseños de investigación para la evaluación de intervenciones en la clínica. Alergia México, 65(2), 178-186. 